\title{
Interactive comment on "Air Quality and Climate Change, Topic 3 of the Model Inter-Comparison Study for Asia Phase III (MICS-Asia III), Part I: overview and model evaluation" by Meng Gao et al.
}

\section{Meng Gao et al. \\ mgao2@seas.harvard.edu}

Received and published: 23 December 2017

The comment was uploaded in the form of a supplement:

https://www.atmos-chem-phys-discuss.net/acp-2017-731/acp-2017-731-AC1-

supplement.pdf 